\title{
Automatic Detection of Glaucoma in Retinal Fundus Images through Image Processing and Data Mining Techniques
}

\author{
R. Geetha Ramani, PhD \\ Department of IST \\ CEG, Anna University \\ Chennai, India
}

\author{
Sugirtharani S. \\ Department of IST \\ CEG, Anna University \\ Chennai, India
}

\author{
Lakshmi B. \\ Department of IST \\ CEG, Anna University \\ Chennai, India
}

\begin{abstract}
Computational techniques are highly used in medical image analysis to aid the medical professionals. Glaucoma is a sight threatening retinal disease that needs attention at its early stages, though it does not reveal any symptoms. Glaucoma is identified usually through cup to disc ratio and ISNT rule. This work involves segmentation of blood vessels, segmentation of optic disc through proposed maximum voting of three segmentation algorithms (K-Means, Wavelet and Histogram based), segmentation of optic cup through intensity thresholding, feature extraction from these segmented structures, feature selection to identify siginificant features, hybrid model involving Naive Bayes to remove noise in data followed by ensemble classification of Reduced Error Pruning Tree. Optic disc segmentation methodology attains an average accuracy of $99.33 \%$. Glaucoma detection accuracy reaches a maximum of $96.42 \%$.
\end{abstract}

\section{General Terms}

Medical image analysis, Image Processing, Data Mining

\section{Keywords}

Glaucoma, Retina, Fundus Image, Optic disc, Maximum voting, hybrid classification

\section{INTRODUCTION}

Computational techniques have great impact in the field of Medicine and Biology. These techniques help the medical practitioners to diagnose any abnormality in advance and provide fruitful treatment. Retinal image analysis has been an ongoing area of research. Automated retinal image analysis aid the ophthalmologists in detecting abnormalities in the retinal structures namely optic disc, blood vessels, macula and fovea, thus diagnosing sight threatening retinal diseases such as Glaucoma and Retinopathy [1]. Glaucoma is the major cause of blindness in working population. Glaucoma is characterised by increased intra-ocular pressure inside the eye leading to changes in the optic disc and optic nerve. It does not reveal its symptoms until later stage. Hence, regular screening of the patients is required to identify the disease, thus demanding high labor, time and expertise. Thus, computational techniques are sought for their analysis.

In this work, identification of Glaucoma is carried out through computational techniques namely image processing and data mining [2]. As the changes in the profile of optic disc act as a biomarker [3] for the onset of the disease, optic disc is segmented through image processing techniques. Optic disc is the brightest part portrayed as oval structure in the retinal fundus image. It encompasses optic cup, which is the brightest central part, optic rim, the surrounding pale part and the blood vessels. All these structures are segmented and their properties are elicited. Then, properties of the disc, cup and blood vessels within optic disc are mined to design a learning model for prediction of Glaucoma.

The remaining of the paper is structured as follows: Section 2 presents the literature survey in automatic diagnosis of Glaucoma, Section 3 highlights the proposed methodology for optic disc segmentation and detection of Glaucoma, Section 4 discusses the results and Section 5 concludes the paper.

\section{LITERATURE SURVEY}

Glaucoma is one of the major causes for blindness. Automated identification of Glaucoma can be of great help to the Ophthalmologists and the society. The existing approaches towards Glaucoma diagnosis is concisely presented here. Generally, the process of Glaucoma detection involves the extraction of optic disc and cup followed by elicitation of its properties such as cup to disc ratio and ISNT ratio to distinguish normal images from Glaucoma affected images. Some of the approaches are briefed here.

In 2009, Nayak et al [4] used Neural networks with Cup to dosc ratio and ISNT ratio, computed from the extracted optic disc and blood vessels as features and achieved a sensitivity and specificity of $100 \%$ and $80 \%$ respectively.

In 2011, Acharya et al [5] incorporated texture and Higher Order Spectra features after z-score normalization and feature selection, and classified with a random-forest classifier, correctly identifying the glaucoma images with an accuracy of more than 91\%. Again in 2011, Ganesh Babu and Shenbagadevi [6] proposed the usage of K means clustering for extraction of optic disc and hence in CDR calculation revealing 90\% match with clinical CDR. Yet another attempt in 2011, Ho et.al, [7] proposed a system that involved vessel detection, vessel in painting, CDR calculation and neuroretinal rim for ISNT rule. K-Nearest Neighbor, SVM and Bayes Classifier with CDR and ISNT ratio yielded a classification accuracy of $95 \%$.

In 2014, GeethaRamani et al [8] proposed a framework based on image features to detect Glaucoma. The methodology incorporated Conversion to various color spaces, channel extraction, statistical, histogram, GLCM based feature extraction and classification through Grafted $\mathrm{C} 4.5$ yielding an accuracy of $86.67 \%$ on HRF images with cross valiation of 3 folds. Again in 2014, Vijapur [9] proposed a data driven workflow for detection of Glaucoma through extraction of energy features from detailed co-efficient images obtained through application of daubechies, symlets and bioorthogonal wavelet filters and computation of cup to disc ratio feature through optic disc attained through disc prediction and cup 
through watershed segmentation attaining a detection accuracy of $96 \%$ on images obtained from K.L.E.Dr.Prabhakar Kore Hospital and Medical Research Center, Belgaum, India.

In 2016, Salem et al [10] attempted to detect Glaucoma through Cup to disc ratio, texture and intensity based features. The predictions from cup to disc ratio and combination of texture and intensity features are correlated to categorize the image as Glaucoma, suspect or non-Glaucoma. The performance of the system reached a sensitivity and specificity of $100 \%$ and $87 \%$ respectively. In 2017, Prakash and Selvathi [11] putforth a morphology based methodology for segmentation of optic disc and optic cup. Optic disc extraction involves extraction of red channel, morphological closing followed by morphological opening and subtraction of opened and closed image. Optic cup segmentation involves independent component analysis on the red channel image, followed by application of morphological closing and thresholding. Subtraction of obtained optic disc and cup reveals the neuro rim region. The method could achieve an accuracy of $99.30 \%$ in optic disc segmentation of HRF images. Then Enhance Local Binary Pattern and GreyLevel Co-Occurence Matrix features were extracted and application of SVM classification was done to categorize Glaucoma and healthy images.

From the previous studies, Glaucoma detection can be performed through domain based features such as cup to disc ratio and ISNT ratio and/or other features such as texture and intensity based features. In the proposed work, domain based and statistical and texture features are extracted from the segmented optic disc, cup and blood vessels and classified to detect Glaucoma. The following section highlights the proposed methodology.

\section{PROPOSED METHODOLOGY}

Automatic Identification of Glaucoma plays a major role in retinal image analysis [12] as it could prevent vision loss in many patients when detected at the appropriate time. The proposed methodology consists of two phases namely image processing phase and data mining phase. The proposed framework is depicted in Figure 1. The modules in each phase are detailed in the following sub-sections.

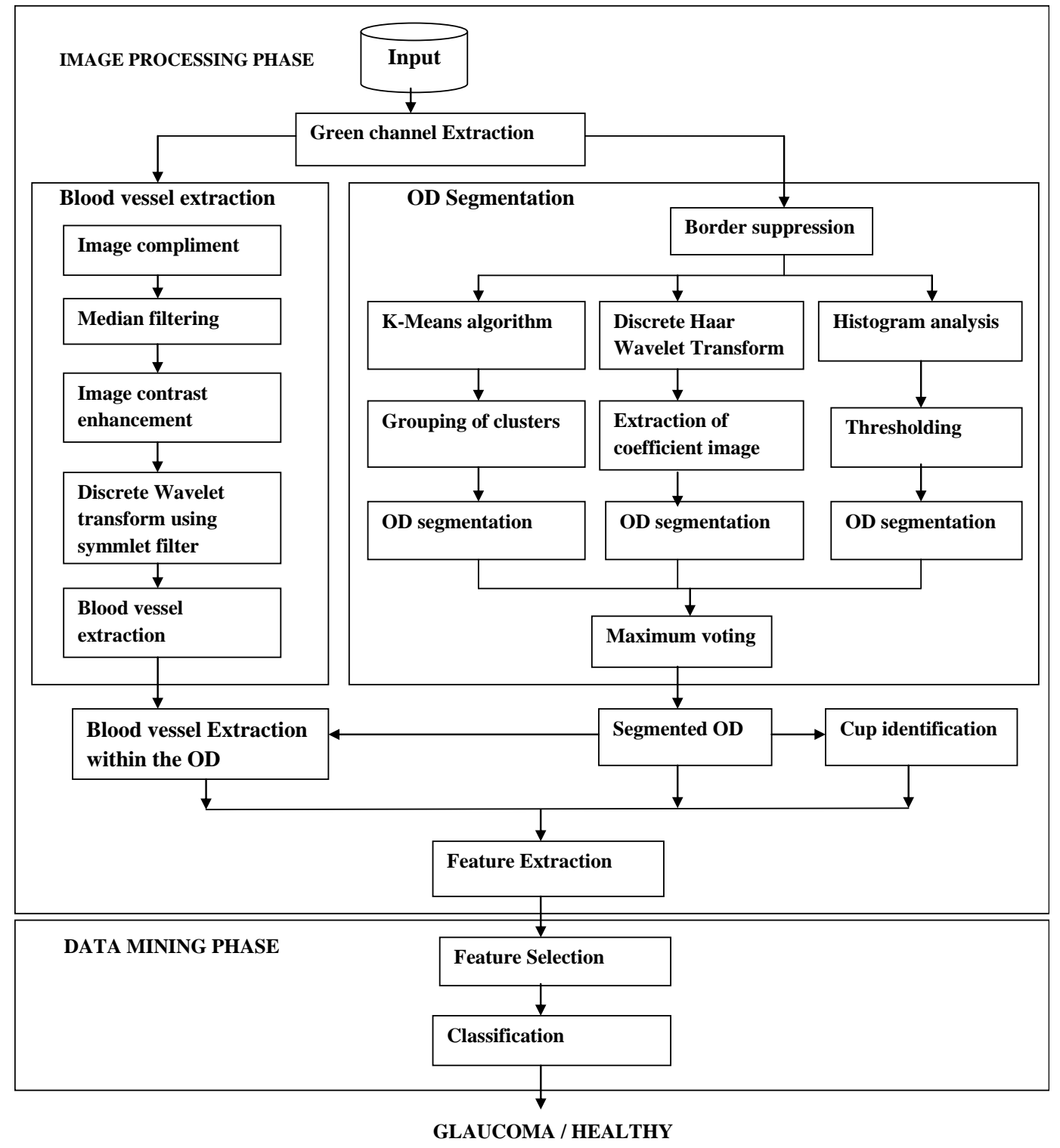

Figure 1: Proposed Framework for Glaucoma Detection 


\subsection{Image Processing Phase}

The image processing phase includes Green channel extraction followed by two segments namely blood vessel segmentation and optic disc segmentation. Blood vessel segmentation involves image compliment, Median filtering, contrast enhancement and Wavelet Transformation through symmlet filters [13]. Optic Disc extraction involves border suppression, optic disc segmentation through K-Means Clustering [14][15], Optic Disc Segmentation through Haar Wavelet Transformation [16] and Optic Disc Segmentation through Histogram analysis [17] followed by maximum voting to delineate final Optic disc boundary. After optic disc extraction, the optic cup is elicited through maximum intensity thresholding. The optic disc is also used to extract the blood vessels within the optic disc. After extraction of optic disc, optic cup and blood vessels within optic disc, the properties of these structures are retrieved forming the feature extraction step. The extracted features are given as input to the data mining phase.
Initially, the input RGB image is converted into Green channel image as it reveals better contrast between the retinal structures. The green channel image serves as input to both the blood vessel and optic disc extraction segments. During blood vessel extraction, the green channel image is complemented. Taking image complement involves subtracting the pixel value from the maximum intensity value (255) so that in the resultant, darker structures would appear bright and bright structures would appear dark. Then, median filtering is performed to remove noise. Median filtering replaces the value of each pixel with median of the surrounding window. After filtering, the contrast of the image is enhanced through Contrast Limited Adaptive Histogram Equalisation (CLAHE) [18], so that the contrast between the vessels and the other structures are prominent making the blood vessel extraction effective. Then, Wavelet Transformation with symmetry filters is applied to extract the blood vessels. The Sample of RGB input image, Green channel image and extracted blood vessels are shown in Figure 2.

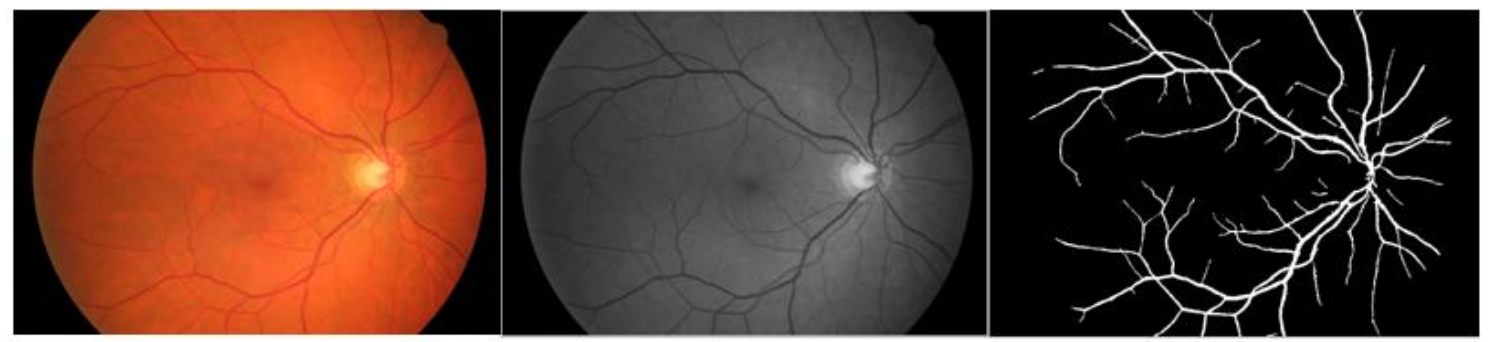

Figure 2: Sample image of RGB, Green channel and extracted blood vessels

During optic disc extraction, border suppression is performed on the green channel image such that the light structures around the bright optic disc are suppressed. Then the border suppressed image is provided as input to three algorithms viz., K-Means clustering, Haar Wavelet Transform and Histogram analysis. K-Means clustering [19] is performed on the border suppressed image. Five clusters are derived from the process. K-Means clustering utilises the Euclidean distance metric to group the similar pixels. On analysing the output of five clusters, the optic disc could be arrived by grouping clusters 3 , 4, and 5. Hence After performing K-Means clustering, the three clusters are grouped to yield the segmented optic disc $\left(\mathrm{Od}_{\mathrm{d}}\right)$. Then, the border suppressed image is provided as input to Haar Wavelet Transformation to yield the optic disc. Out of the four output responses (approximate and detailed images),the approximate image is further considered for processing. The image is then binaries to obtain the optic disc $\left(\mathrm{Od}_{\mathrm{d}}\right)$. Then, the histogram of green channel image is analysed. The peak at the brightest side is spotted and used to segment the optic disc. This procedure yields a segmented optic disc $\left(\mathrm{Ou}_{\mathrm{dh}}\right)$.

The three segmented OD images are analysed to obtain the maximum voting. The pixels that have been regarded as optic disc in at least two algorithms are finalised as optic disc pixel in the final segmentation of optic disc. The optic disc boundary is then adjusted to form a regular ellipse. Sample of K-Means segmented optic disc, Haar wavelet segmented optic disc, histogram based optic disc and the final segmented optic disc is portrayed in Figure 3.
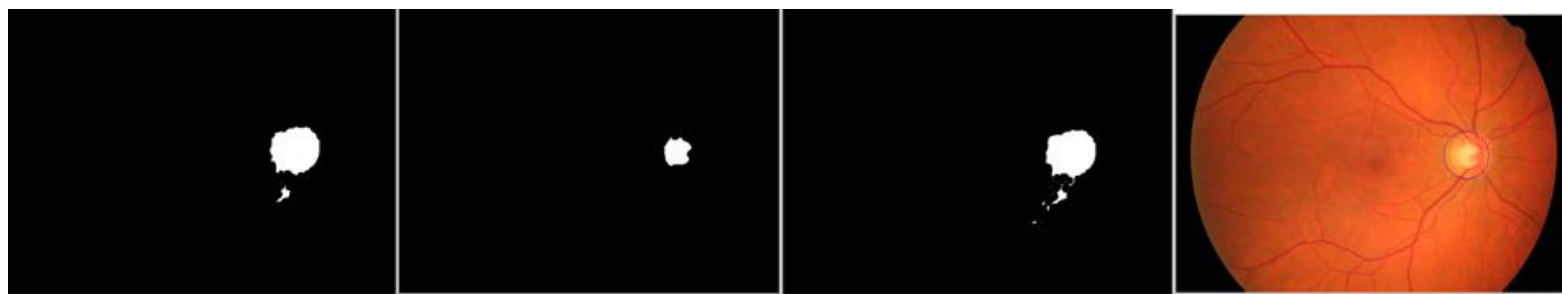

Figure 3: Outcome of K-means, Haar Wavelet, Histogram analysis and final segmentation

After the extraction of optic disc, the optic cup is delineated from the disc based on the intensity thresholding. The obtained optic disc is imposed on green channel image and the maximum intensity poxels are regarded as cup. Sample of the RGB image with detected OD and cup boundary is shown in Figure 5.

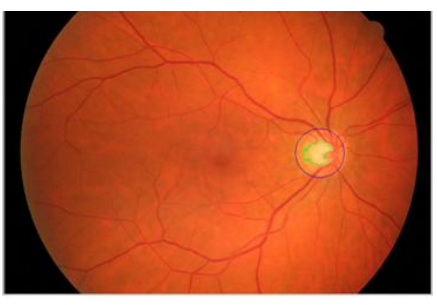

Figure 4: RGB image showing plot of segmented optic disc and cup 
Having extracted the optic disc, optic cup and blood vessels within the optic disc, their properties are elicited.

The features extracted from binary image of optic disc and optic cup include disc area, cup area, disc diameter, cup diameter, cup to disc ratio of diameter, and cup to disc ratio of area The features extracted from binary image of blood vessels within optic disc include number of vessel pixels in inferior, superior, Nasal and temporal regions, the truth condition of is inferior greater than superior, is inferior greater than nasal, is inferior greater than temporal, is superior greater than nasal, is superior greater than temporal, and is nasal greater than temporal. The features [19][20] extracted from the optic disc of green channel image include entropy, mean, range, standard deviation, contrast, correlation, energy, homogeneity, median, mode, autocorrelation, cluster prominence, cluster shade, difference in entropy, difference variance, dissimilarity, information measre 1 of correlation, information measure 2 of correlation, inverse difference, maximum probability, sumof average, sum of entropy, sum of square variance and sum of variance, The features extracted from optic cup of green channel image include cup contrast, cup entropy, cup homogeneity, cup mean, cup standard deviation and cup sum. Thus totally 47 features are extracted from the segmented structures for setection of Glaucoma. These features form the input to data mining phase. The modules in the data mining phase are described in the next sub-sectio

\subsection{Data Mining Phase}

The features extracted from the image mining phase are provided as input to the data mining phase. The data mining phase includes feature selection and classification. These procedures are discussed here.

Features selection procedure selects the significant features for the problem in hand. The aignificant features aids in improving the categorisation of Glaucoma. The reduced number of features also reduces the complexity in the further processing. Different feature selection [21] procedures were attempted. They include Correlation based [22], Chisquared based, Gain Ratio based, Information gain based feature selection. It was seen that all feature selection algorithms perform better than suring classification without application of feature selection.
The selected features are given as input to classification process. Classification takes the training samples and builds a model so that any instance without class label could be labelled. Different classifiers were attempted to categorise Glaucoma and Healthy images. Usually, a very few instances of the dataset drag the accuracy of the learning model as they act as outliers. Hence a hybrid model [23] that employs Naive Bayes followed by classification is utilised. Here, the instances are first classified using Naive Bayes, the misclassifications are removed, then various classification procedures are tried to classify the remaining instances. It is also noticed that prediction from bagged classifiers are better when compared to prediction of single classifier. Various classifiers [24] viz. Random Tree (RT), Aleternating Decision Tree (ADT), C4.5, Reduced Error Pruning Tree (REP) were attempted.

Thre performance of the classification algorithms is evaluated using 10 fold cross validation, where the data is divided into ten folds with first nine folds as training data and the other fold as testing data. The procedure is repeated for ten iterations with each fold taking different roles in each iteration. The classification is then aggregated to project the

performance of the classifier. The following section discusses the experimental setup and results in detail.

\section{RESULTS AND DISCUSSION}

The proposed framework is evaluated on HRF [25] image dataset. The dataset consists of 30 images, out of which 15 are normal images and 15 are Glaucoma affected images. The implementation of the proposed work was dome through Matlab 2013a and Weka. The steps in image processing phase were carried out in Matlab and the data mining steps were performed in Weka. The results first projects the efficiency of optic disc segmentation. Then, results show the Glaucoma detection without eliminating instance with Naive Bayes. Then the classification accuracy obtained after eliminating instances through Naive Bayes are reported. The classification results are reported on features with and without feature selection.

Primarilly, the optic disc is segmented through the proposed method. Table 1 reports the efficiency of optic disc segmentation through Euclidean distance between the centroids, accuracy, sensitivity, specificity, Positive Predictive Value, Dice Score and Jaccard Index.

Table 1: Optic disc segmentation results

\begin{tabular}{|l|l|l|l|l|l|l|l|}
\hline Image & $\begin{array}{l}\text { Euclidean } \\
\text { Distance }\end{array}$ & Accuracy & Sensitivity & Specificity & PPV & Dice & Jaccard \\
\hline 01_g & 38 & 99.44 & 87.96 & 99.58 & 73.26 & 0.7994 & 0.6658 \\
\hline 01_h & 92 & 99.45 & 82.44 & 99.72 & 82.02 & 0.8223 & 0.6982 \\
\hline 02_g & 20 & 99.17 & 76.63 & 99.48 & 66.25 & 0.7106 & 0.5511 \\
\hline 02_h & 70 & 99.00 & 65.98 & 99.55 & 71.34 & 0.6855 & 0.5215 \\
\hline 03_g & 55 & 99.62 & 82.49 & 99.93 & 95.29 & 0.8843 & 0.7925 \\
\hline 03_h & 91 & 99.57 & 90.80 & 99.72 & 85.07 & 0.8784 & 0.7831 \\
\hline 04_g & 48 & 99.46 & 83.60 & 99.70 & 80.71 & 0.8213 & 0.6968 \\
\hline 04_h & 94 & 99.57 & 88.20 & 99.76 & 86.08 & 0.8712 & 0.7719 \\
\hline 05_g & 36 & 99.08 & 72.85 & 99.44 & 63.67 & 0.6795 & 0.5145 \\
\hline 05_h & 101 & 99.48 & 79.02 & 99.84 & 89.59 & 0.8397 & 0.7237 \\
\hline 06_g & 47 & 99.14 & 72.91 & 99.53 & 69.68 & 0.7126 & 0.5535 \\
\hline 06_h & 66 & 99.20 & 77.59 & 99.49 & 67.48 & 0.7218 & 0.5647 \\
\hline 07_g & 68 & 99.67 & 92.22 & 99.77 & 85.44 & 0.8870 & 0.7969 \\
\hline 07_h & 75 & 99.06 & 68.51 & 99.55 & 71.27 & 0.6986 & 0.5368 \\
\hline 08_g & 66 & 99.48 & 85.99 & 99.66 & 79.24 & 0.8248 & 0.7018 \\
\hline 08_h & 90 & 99.09 & 70.60 & 99.53 & 69.89 & 0.7024 & 0.5413 \\
\hline
\end{tabular}




\begin{tabular}{|l|l|l|l|l|l|l|l|}
\hline 09_g & 66 & 99.25 & 81.76 & 99.46 & 65.47 & 0.7271 & 0.5712 \\
\hline 09_h & 74 & 99.46 & 82.26 & 99.72 & 82.23 & 0.8225 & 0.6984 \\
\hline 10_g & 27 & 99.56 & 92.47 & 99.65 & 77.87 & 0.8454 & 0.7322 \\
\hline 10_h & 59 & 99.33 & 80.68 & 99.60 & 74.32 & 0.7737 & 0.6309 \\
\hline 11_g & 38 & 99.25 & 89.77 & 99.40 & 70.91 & 0.7924 & 0.6561 \\
\hline 11 h & 40 & 99.12 & 73.12 & 99.50 & 67.74 & 0.7033 & 0.5423 \\
\hline 12_g & 84 & 99.36 & 82.56 & 99.60 & 74.13 & 0.7812 & 0.6409 \\
\hline 12_h & 33 & 99.42 & 85.59 & 99.61 & 74.83 & 0.7985 & 0.6646 \\
\hline 13_g & 15 & 99.35 & 99.08 & 99.35 & 62.44 & 0.7661 & 0.6208 \\
\hline 13_h & 32 & 99.31 & 84.09 & 99.50 & 68.10 & 0.7526 & 0.6033 \\
\hline 14_g & 168 & 98.93 & 72.11 & 99.22 & 49.39 & 0.5863 & 0.4147 \\
\hline 14_h & 47 & 99.39 & 88.85 & 99.52 & 68.79 & 0.7754 & 0.6332 \\
\hline 15_g & 84 & 99.32 & 77.59 & 99.66 & 78.35 & 0.7797 & 0.6389 \\
\hline 15_h & 45 & 99.55 & 95.15 & 99.60 & 74.50 & 0.8357 & 0.7177 \\
\hline Mean & $\mathbf{6 2 . 3}$ & $\mathbf{9 9 . 3 3}$ & $\mathbf{8 2 . 0 9}$ & $\mathbf{9 9 . 5 9}$ & $\mathbf{7 4 . 1 8}$ & $\mathbf{0 . 7 7 6 0}$ & $\mathbf{0 . 6 3 9 3}$ \\
\hline
\end{tabular}

From the table, it is observed that the proposed optic disc segmentation algorithm performs comparably for utilisation in detection of Glaucoma. After extracting the optic disc, optic cup and blood vessels are extracted and their features were elicited. Detection of Glaucoma with the extracted features with various features are presented in Table 2. Table 2 reports the accuracy of categorisation of Glaucoma and healthy images.

Table 2: Performance of Classifiers in detecting Glaucoma

\begin{tabular}{|l|l|l|l|l|l|}
\hline Classifier & $\begin{array}{l}\text { Without } \\
\text { feature } \\
\text { selection }\end{array}$ & CFS & $\begin{array}{l}\text { CHI } \\
\text { SQUARED }\end{array}$ & $\begin{array}{l}\text { GAIN } \\
\text { RATI } \\
\text { O }\end{array}$ & $\begin{array}{l}\text { INFO } \\
\text { GAIN }\end{array}$ \\
\hline REP & 76.66 & 73.33 & 73.33 & 73.33 & 73.33 \\
\hline C4.5 & 63.33 & 70.00 & 70.00 & 70.00 & 70.00 \\
\hline NB & 80.00 & 83.33 & 83.33 & 83.33 & 83.33 \\
\hline ADT & 73.33 & 80.00 & 76.66 & 76.66 & 76.66 \\
\hline RT & 66.66 & 76.66 & 63.33 & 76.66 & 76.66 \\
\hline
\end{tabular}

The results from Table 2 reveal that the accuracy of categorisation should be improved for detection of Glaucoma. As prediction from bagged classifiers usually yield better prediction than single classifier, classification was attempted through bagged classifiers. The following table 3 presents the classification accuracy of bagged classifiers.

Table 3: Performance of Bagged classifiers in detecting Glaucoma

\begin{tabular}{|l|l|l|l|l|l|}
\hline $\begin{array}{l}\text { Bagged } \\
\text { Classifier }\end{array}$ & $\begin{array}{l}\text { Without } \\
\text { feature } \\
\text { selection }\end{array}$ & CFS & $\begin{array}{l}\text { CHI } \\
\text { SQUARED }\end{array}$ & $\begin{array}{l}\text { GAIN } \\
\text { RATIO }\end{array}$ & $\begin{array}{l}\text { INFO } \\
\text { GAIN }\end{array}$ \\
\hline $\begin{array}{l}\text { Bagged } \\
\text { REP }\end{array}$ & 83.33 & 83.33 & 80.00 & 80.00 & 76.66 \\
\hline $\begin{array}{l}\text { Bagged } \\
\text { C4.5 }\end{array}$ & 76.66 & 73.33 & 73.33 & 73.33 & 73.33 \\
\hline $\begin{array}{l}\text { Bagged } \\
\text { NB }\end{array}$ & 76.66 & 83.33 & 83.33 & 83.33 & 83.33 \\
\hline $\begin{array}{l}\text { Bagged } \\
\text { ADT }\end{array}$ & 73.33 & 76.66 & 76.66 & 76.66 & 73.33 \\
\hline $\begin{array}{l}\text { Bagged } \\
\text { RT }\end{array}$ & 73.3 & 76.66 & 73.33 & 73.33 & 70.00 \\
\hline
\end{tabular}

From table 3, it is found that bagging improves the classification outcomes. But the accuracy has to be still improved for practical purposes. On analysis of the data, the training accuracy itself was lower as two instances were not correctly fitting any rule. Hence the two instances were removed using naive Bayes classifier. Then after removal of two instances, considering it as outlier, the bagged classifiers were attempted to classify the remaining instances. The table
4 reports the improved results obtained through this procedure.

Table 4: Outcome of bagged classifiers on dataset with eliminated instances through Naive Bayes

\begin{tabular}{|l|l|l|l|l|l|}
\hline $\begin{array}{l}\text { Bagged } \\
\text { Classifier }\end{array}$ & $\begin{array}{l}\text { Without } \\
\text { feature } \\
\text { election }\end{array}$ & CFS & $\begin{array}{l}\text { CHI } \\
\text { SQUARED }\end{array}$ & $\begin{array}{l}\text { GAIN } \\
\text { RATIO }\end{array}$ & $\begin{array}{l}\text { INFO } \\
\text { GAIN }\end{array}$ \\
\hline $\begin{array}{l}\text { Bagged } \\
\text { REP }\end{array}$ & 89.28 & 96.42 & 96.42 & 96.42 & 96.42 \\
\hline $\begin{array}{l}\text { Bagged } \\
\text { C4.5 }\end{array}$ & 89.28 & 92.45 & 89.28 & 89.28 & 92.85 \\
\hline $\begin{array}{l}\text { Bagged } \\
\text { NB }\end{array}$ & 89.28 & 92.85 & 89.28 & 89.28 & 89.28 \\
\hline $\begin{array}{l}\text { Bagged } \\
\text { NB }\end{array}$ & 85.71 & 89.28 & 89.28 & 85.71 & 89.28 \\
\hline $\begin{array}{l}\text { Bagged } \\
\text { RT }\end{array}$ & 89.28 & 92.85 & 92.85 & 89.28 & 89.28 \\
\hline
\end{tabular}

The results in the table 4 reveal satisfactory prediction of the disease. The best results are achieved when the feature selection was employed, classification through Naive Bayes and removal of outliers followed by bagged classification through REP Tree, reporting $96.42 \%$. The proposed system can act as a decision support system for ophthalmologists in detecting Glaucoma.

\section{CONCLUSION}

Glaucoma is a major cause of blindness in the society. Computational techniques are sought for detecting Glaucoma. This work involves extraction of blood vessels through symmlet wavelet transformation, extraction of optic disc through maximum voting of three segmentation algorithms, cup segmentation through intensity thresholding, extraction of blood vessels within the optic disc, elicitation of features associated with these structures, feature selection, classification through hybrid model involving Naive Bayes followed by ensemble classification of Reduced Error Pruning tree reporting an accuracy of $96.42 \%$. The methodology serves the society.

\section{REFERENCES}

[1] Han, J., Pei, J. and Kamber, M., 2011. Data mining: concepts and techniques. Elsevier.

[2] Ramani, R.G., Lakshmi, B. and Jacob, S.G., 2012. Automatic prediction of Diabetic Retinopathy and Glaucoma through retinal image analysis and data mining techniques. In Machine Vision and Image Processing (MVIP), 2012 International Conference on (pp. 149-152). IEEE. 
[3] Glaucoma Tutorial (available at) http://www.opticdisc.org/tutorials/glaucoma_evaluation_basics/page13.ht $\mathrm{ml}$

[4] Nayak, J., Acharya, R., Bhat, P.S., Shetty, N. and Lim, T.C., 2009. Automated diagnosis of glaucoma using digital fundus images. Journal of medical systems, 33(5), p.337.

[5] Acharya, U.R., Dua, S., Du, X. and Chua, C.K., 2011. Automated diagnosis of glaucoma using texture and higher order spectra features. IEEE transactions on Information Technology in Biomedicine, 15(3), pp.449455 .

[6] Babu, T.G. and Shenbagadevi, S., 2011. Automatic detection of glaucoma using fundus image. European Journal of Scientific Research, 59(1), pp.22-32.

[7] Ho, C.Y., Pai, T.W., Chang, H.T. and Chen, H.Y., 2011. An atomatic fundus image analysis system for clinical diagnosis of glaucoma. In Complex, Intelligent and Software Intensive Systems (CISIS), 2011 International Conference on (pp. 559-564). IEEE.

[8] GeethaRamani, R, Dhanapackiam, C and Lakshmi, B. 2013. Automatic Detection of Glaucoma in Fundus Images through Image Features, International conference on Knowledge Modelling and Knowledge Management, pp. 135-144.

[9] Vijapur, N., Srinivasa, R. and Rao, K., 2014. Improved Efficiency of Glaucoma Detection by using Wavelet Filters Prediction and Segmentation Method. International journal of electronics, Electrical and Computational System, 3(8).

[10] Salam, A.A., Khalil, T., Akram, M.U., Jameel, A. and Basit, I., 2016. Automated detection of glaucoma using structural and non structural features. SpringerPlus, 5(1), p.1519.

[11] Prakash, N.B. and Selvathi, D., 2017. An Efficient Detection System for Screening Glaucoma in Retinal Images. Biomedical and Pharmacology Journal, 10(1), pp.459-465.

[12] Ramani, R.G. and Lakshmi, B., 2015. Automatic retinal vessel segmentation through Gabor filtering, principal component analysis and ensemble of classifiers (C4. 5 with bagging). Advances in Natural and Applied Sciences, 9(6 SE), pp.600-607.

[13] Hariprasath, S. and Mohan, V., 2009. Iris pattern recognition using complex wavelet and wavelet packet transform. Journal of Computer Applications, 2(2).
[14] Lloyd, S., 1982. Least squares quantization in PCM. IEEE transactions on information theory, 28(2), pp.129137.

[15] GeethaRamani, R. and Lakshmi, B., 2016. Retinal blood vessel segmentation employing image processing and data mining techniques for computerized retinal image analysis. Biocybernetics and Biomedical Engineering, 36(1), pp.102-118.

[16] Lai, Y.K. and Kuo, C.C.J., 1997, October. Image quality measurement using the Haar wavelet. In Optical Science, Engineering and Instrumentation'97 (pp. 127-138). International Society for Optics and Photonics.

[17] Dehghani, A., Moghaddam, H.A. and Moin, M.S., 2012. Optic disc localization in retinal images using histogram matching. EURASIP Journal on Image and Video Processing, 2012(1), p.19.

[18] Pizer, S.M., Johnston, R.E., Ericksen, J.P., Yankaskas, B.C. and Muller, K.E., 1990, May. Contrast-limited adaptive histogram equalization: speed and effectiveness. In Visualization in Biomedical Computing, 1990., Proceedings of the First Conference on (pp. 337-345) IEEE.

[19] Geetha Ramani, R. and Lakshmi, B., 2013. Multi-class classification for prediction of retinal diseases (retinopathy and occlusion) from fundus images Proceedings of ICKM, 13, pp.122-134.

[20] Haralick, R.M. and Shanmugam, K., 1973. Textural features for image classification. IEEE Transactions on systems, man, and cybernetics, 3(6), pp.610-621.

[21] Hall, M.A., 1999. Correlation-based feature selection for machine learning (Doctoral dissertation, The University of Waikato).

[22] Tang, J., Alelyani, S. and Liu, H., 2014. Feature selection for classification: A review. Data Classification: Algorithms and Applications, p.37.

[23] Ramani, R.G., Lakshmi, B. and Alaghu Meenal, A., 2015. A hybrid classification model employing Genetic algorithm and Root Guided Decision Tree for improved categorization of data. ARPN Journal of Engineering and Applied Sciences, 10(21), pp.9968-9975.

[24] Zhao, Y. and Zhang, Y., 2008. Comparison of decision tree methods for finding active objects. Advances in Space Research, 41(12), pp.1955-1959.

[25] GOLD standard database (available at) https://www5.cs.fau.de/research/data/fundus-images/ 